\title{
Effect of prolonged submaximal exercise on serum oxidative stress biomarkers (d-ROMs, MDA, BAP) and oxidative stress index in endurance horses
}

\author{
Nika Brkljača Bottegaro ${ }^{1 *}$ (D) Jelena Gotić ${ }^{2}$, Jelena Šuran ${ }^{3}$, Diana Brozić ${ }^{4}$, Karla Klobučar ${ }^{5}$, Krunoslav Bojanić ${ }^{6}$
} and Zoran Vrbanac ${ }^{7}$

\begin{abstract}
Background: Oxidative stress (OS) associated with an intense exercise may have a negative influence on equine health. The aim of this study was to determine the effects of endurance races on oxidative and antioxidative status of horses by evaluating changes in reactive oxygen metabolites (d-ROMs), malondialdehyde (MDA), biological antioxidant potential (BAP) and oxidative stress index (OSI) values. The study was carried out on 53 race starts (28 individual horses) competing at different endurance races according to distance (40 and $80 \mathrm{~km}$ ) and difficulty (easy and demanding). Blood samples were taken before and after the race.

Results: Compared to levels of OS serum biomarkers before the race, an increase in values of $d$-ROMs $(P<0.01)$, MDA $(P<0.01)$, and BAP $(P<0.001)$, and a decrease in OSI $(P<0.001)$ have been noted after the race. Contrary to other measured biomarkers, BAP did not show significant individual effects of horses. Horses competing at shorter races have shown a significant change in $\mathrm{d}-\mathrm{ROMs}(P=0.002)$, BAP $(P<0.001)$ and OSI $(P=0.004)$, whereas those competing at longer races in MDA $(P=0.002), \operatorname{BAP}(P<0.001)$ and OSI $(P<0.001)$ post-race values. Endurance racing induced changes in values of $\mathrm{d}-\mathrm{ROMS}, \mathrm{BAP}$ and $\mathrm{OSI}$ during both easy and demanding races.

Conclusions: Changes in all measured OS biomarkers indicate that prolonged aerobic exercise during endurance race could contribute to the imbalance between oxidants and antioxidants in horses, mainly characterised by a pronounced antioxidant response. Biological antioxidant potential was found to be the most reliable biomarker of OS in endurance horses in the present study.
\end{abstract}

Keywords: Horse, Oxidative stress, Reactive oxygen metabolites, Malondialdehyde, Biological antioxidant potential, Oxidative stress index, Endurance race

\section{Background}

Endurance riding is an extremely demanding equine discipline since horses compete over distances of up to $160 \mathrm{~km}$ in a day. An endurance ride is divided into several phases of 20 to $40 \mathrm{~km}$, each followed by a mandatory resting period. Before the ride, as well as after each phase, horses undergo a mandatory veterinary examination and can be eliminated if

\footnotetext{
* Correspondence: nikabottegaro@gmail.com

${ }^{1}$ Clinic for Surgery, Orthopaedics and Ophthalmology, Faculty of Veterinary

Medicine, University of Zagreb, Heinzelova 55, 10000 Zagreb, Croatia

Full list of author information is available at the end of the article
}

their metabolic status or orthopaedic condition are found inadequate to enable them to continue the ride.

Exercise induces increased oxygen consumption which in return affects the oxidant/antioxidant status [5]. During short-term maximal exercise in Thoroughbred racehorses, there is an increase in oxygen consumption that reaches levels 30 times above basal [1]. Even though endurance riding is classified as a prolonged aerobic exercise [2], the high oxygen demands over a longer period of time result in an increased reactive oxygen species (ROS) formed by 1 to $2 \%$ of the oxygen that is not completely reduced into carbon dioxide and water [3]. The consequence of

(c) The Author(s). 2018 Open Access This article is distributed under the terms of the Creative Commons Attribution 4.0 International License (http://creativecommons.org/licenses/by/4.0/), which permits unrestricted use, distribution, and reproduction in any medium, provided you give appropriate credit to the original author(s) and the source, provide a link to the Creative Commons license, and indicate if changes were made. The Creative Commons Public Domain Dedication waiver (http://creativecommons.org/publicdomain/zero/1.0/) applies to the data made available in this article, unless otherwise stated. 
increased oxidants is a disarranged equilibrium between oxidants and antioxidants resulting in oxidative stress (OS) [4]. Apart from chronic accumulation of ROS, OS can also be a sequel of insufficient antioxidant defence systems, causing damage to all cell components, especially DNA, lipids, and proteins [5].

OS is characterised by changes in different biomarkers but the determination of reactive oxygen metabolites (d-ROMs), an indirect method evaluating the free radicals in the serum, is considered a 'gold standard' biomarker for measuring total systemic oxidative status [6]. Among other OS biomarkers, the biological antioxidant potential (BAP) test indicates systemic antioxidative properties and reflects the reduction potential in the serum [7], whereas malondialdehyde (MDA) is one of the better-known secondary products of lipid peroxidation [8] used as an indicator of cell membrane injury [9]. The oxidative stress index (OSI) is an arbitrary parameter derived from values of d-ROMs and BAP to represent an individual's antioxidant potential against the condition of OS [10]. In equine medicine, OSI has been investigated in resting Thoroughbred foals [11], Thoroughbred racehorses [12], and Thoroughbred horses after castration [13].

Oxidative stress in horses induced by endurance races has been investigated in a few studies mainly focusing on antioxidant status [14-17]. Oxidative properties have been assessed by measurements of the following biomarkers: lipid hydroperoxides (LPO) $[17,18]$ and total barbituric acid reactive substances (TBARS) $[14,16]$. On the other hand, antioxidant status in endurance horses has been more intensively studied by evaluating several different biomarkers: $\alpha$-tocopherol (TOC) and glutathione (GSH) [15-18]; glutathione peroxidase (GPx) activity [14, 15, 17, 18], glutathione redox ratio [16], glutathione reductase, superoxide dismutase (SOD), and total antioxidans status (TAS) [14].

However, data on changes of d-ROMs and BAP in relation to endurance exercise in horses, to the best of our knowledge, have not been reported. All the mentioned studies [14-18] have been performed on horses competing at only higher levels of exercise, although a vast majority of horses in this sport compete at lower level national endurance races.

The aim of this study was to determine the effects of endurance races on blood levels of d-ROMs, BAP, and MDA OS biomarkers in horses. It was hypothesised that even endurance races at shorter distances induce OS in horses. The second hypothesis was that endurance exercise causes changes in OSI levels which could emphasise a more pronounced prooxidant or antioxidant response.

\section{Methods}

\section{The competition and conditions}

The study included horses competing at four national competitions held from April to July 2017 at different locations in Croatia. The horse owners were offered to participate in the study prior to the first veterinary inspection of their horse (s) at the competition sites. The races differed according to terrain condition and configuration, altitude changes, and weather conditions, thus considering these variations, the races were categorised as demanding or easy. Demanding races were those with a considerable part of rocky racetrack, higher altitude differences and/or with higher temperature and humidity. Two races were classified as easy and two as demanding. Also, there were races of both 40 and $80 \mathrm{~km}$ distances at each competition.

\section{Horses}

All horses competing at the mentioned races were eligible for enrolment in this study. Forty-five horses were enrolled following owner consent. Horses had been transported from varying distances and arrived at the event several hours (at least two) before the first veterinary examination. All the horses participating in the study successfully passed the veterinary inspection prior the start, as well as all the inspections during and after the race. Since the inclusion criterion for horses was to successfully complete the race, only the finisher horses were included in the further study.

The average speed of horses in the study population was also evaluated with a cut-off of $14 \mathrm{~km} / \mathrm{h}$ which is the minimal average speed required for the Federation Equestre International 4* championship qualification according to the FEI Endurance rules 2017.

\section{Sample collection and analysis}

Blood samples $(5 \mathrm{ml})$ were collected by jugular venipuncture into vacuum tubes for serum with gel using a vacutainer (Vacutainer, Becton Dickenson, USA) $1 \mathrm{~h}$ before the start and $30 \mathrm{~min}$ after the end of the race. The samples were left to coagulate for $30 \mathrm{~min}$ and thereafter centrifuged at $3500 \mathrm{pm}$ for $15 \mathrm{~min}$. The samples were shipped in cooling bags at $4{ }^{\circ} \mathrm{C}$ to the laboratory, and within $4 \mathrm{~h}$ after sampling stored at $-80^{\circ} \mathrm{C}$ until further analyses.

Serum levels of d-ROMs and BAP were measured using Diacron $^{\circ}$ commercial kits (Grosseto, Italy) on biochemical analyzer Olympus AU640 (Olympus, Japan) according to manufacturer's instructions. The results of the d-ROMs analysis were expressed in Carratelli units (U.Carr.), where 1 U.Carr. corresponds to $0.8 \mathrm{mg} / \mathrm{L} \mathrm{H}_{2} \mathrm{O}_{2}$. The results of the BAP analysis were expressed in $\mu \mathrm{mol} / \mathrm{L}$ of the reduced ferric ions. The assays used in this study had been previously validated for use in horses $[6,19]$.

Malondialdehyde concentration was determined with a slight modification of a previously reported method [8]. Briefly, a volume of $150 \mu \mathrm{L}$ of serum was added to $50 \mu \mathrm{L}$ of water and $50 \mu \mathrm{L}$ of $\mathrm{NaOH}(3 \mathrm{~N})$. After $30 \mathrm{~min}$ of shaking water bath with an incubation at $60{ }^{\circ} \mathrm{C}, 250 \mu \mathrm{L}$ $6 \%$ phosphoric and $0.8 \%$ thiobarbituric acid were added and the mixture was heated at $90{ }^{\circ} \mathrm{C}$ for $45 \mathrm{~min}$. The 
mixture was cooled and extracted by adding $250 \mu \mathrm{l}$ of pure methanol and $100 \mu \mathrm{l} 10 \%$ of sodium dodecyl sulphate, vortex-mixed for $1 \mathrm{~min}$ and centrifuged at $3000 \times g$ for $10 \mathrm{~min}$. A Shimadzu 2010 LC system equipped with an InertSustain C18 $(4.6 \mathrm{~mm} \times 150 \mathrm{~mm} \times$ $5 \mu \mathrm{m}$ ) column (GL Sciences) and a UV-visible detector was used for analysis with the following conditions: the UV wavelength was set at $532 \mathrm{~nm}$, injection volume of $20 \mu \mathrm{L}$, and the column oven temperature at $38{ }^{\circ} \mathrm{C}$. The mobile phase was as follows: pump A, methanol, and pump B, $50 \mathrm{mM}$ potassium dihydrogen phosphate with $\mathrm{pH}$ of $6.2(50: 50, v / \mathrm{v})$. The flow rate of the mobile phase was $1 \mathrm{~mL} / \mathrm{min}$ with constant flow control. The standard curve was prepared using 1,1,3,3-tetraethoxypropane with the linearity of 0.98 and reliability of $97.5 \%$ for given standard concentration range $(0.5-15.175 \mu \mathrm{M}$ of standard). MDA concentration was expressed as $\mu \mathrm{mol}$ per $\mathrm{L}$ serum. The method was in-house validated for use in horse serum, with the inter- and intra-assay coefficients of variation of less than $5 \%$.

The OSI was calculated from the measured values of $\mathrm{d}-\mathrm{ROMs}$ and BAP using the ratio of $\mathrm{d}-\mathrm{ROMs} / \mathrm{BAP}$ multiplied by 100 [11].

\section{Statistical methods}

All exploratory data analyses and statistical tests were performed using the $\mathrm{R}$ programming language version 3.2.21 [20]. The dependent variables of interest were the changes in values after the race compared to before the race for each OS biomarker. The explanatory variables of interest that were collected from horses (gender and average speed) and race events characteristics (race distance and difficulty) were used to evaluate the changes in values of OS biomarkers within each subpopulation category and for comparisons between the respective subpopulation categories of horses. Horses never ran more than once per event and some horses participated at several events. Therefore, the overall average change (post-race value - pre-race value) was analysed via hierarchical linear mixed-effects regression models due to repeated measurements on the same horse subjects and using linear regression models for analysis of data within each race. Modelling was performed using the lme4 software package [21]. Outliers were assessed using leave-one-out method for mixed models and Bonferroni outlier test for linear models. As no significant influence on model coefficient estimates was observed, the data were kept in the model. The alpha level of significance for model fits and regression coefficients was taken at less than 0.05. Data were expressed as an average ( \pm standard error of mean (SEM)).

\section{Results}

\section{Study population characteristics}

Forty-five horses participated in the study. However, 28 individual horses successfully met the inclusion criteria in 53 race starts, since some horses participated in more races. The characteristics of the study population are presented in the Table 1 . The average age of the studied horses was 7.3 years $( \pm 0.3$ SEM) and their average speed during races was $13.5( \pm 0.2 \mathrm{SEM}) \mathrm{km} / \mathrm{h}$. The average speed was $13.7( \pm 0.3 \mathrm{SEM}) \mathrm{km} / \mathrm{h}$ at $40 \mathrm{~km}$ races and $13.2( \pm 0.3 \mathrm{SEM}) \mathrm{km} / \mathrm{h}$ at $80 \mathrm{~km}$ races. There were no significant differences between the average speed at 40 and $80 \mathrm{~km}$ races $(P=0.3)$. However, there was a significant association $(P<0.001)$ of the average speed and the race difficulty with the average speed of $14.3( \pm 0.2 \mathrm{SEM}) \mathrm{km} / \mathrm{h}$ at easy races and the average speed of $12.3( \pm 0.4$ SEM) $\mathrm{km} / \mathrm{h}$ at demanding races.

\section{Comparison of pre- and post-race values of OS biomarkers}

The values of all oxidative stress markers evaluated in this study have significantly changed after the race compared to the values before the race and their distribution is depicted in Fig. 1. The most significant changes were noted for BAP and OSI $(P<0.001)$. On average, values of BAP, d-ROMs, and MDA increased, while values of OSI decreased after the race. Inspection of random effects of horse subjects showed that the estimates of average change of MDA, d-ROMs, and OSI are extremely variable between and within horses. Ten horses had discordant changes (an increase at one race and a decrease at another) in values of d-ROMs, 11 in MDA and 8 in values of OSI. On the other hand, the individual variation of the subjects was low for BAP.

Values of different OS markers in relation to categories of race distances and difficulty, and of average speed and gender of horses are presented in Tables 2 and 3, respectively. On average, there was a significant change in values of BAP and OSI irrespective of the race distances. However, a significant change in values of d-ROMs was observed only at $40 \mathrm{~km}$ and of MDA only at $80 \mathrm{~km}$ race distances. Values of d-ROMs, BAP and OSI showed significant differences before and after the race for both easy and demanding races. Changes in concentrations of MDA were significantly different than before the race

Table 1 Characteristics of the study population of horses attending four endurance races

\begin{tabular}{ll}
\hline Descriptive variables & Study population \\
\hline Horses & 28 individuals \\
Breed (Arabian / other breeds) & $14 / 14$ \\
Gender (females / males) & $18 / 10$ \\
Races & 53 records \\
Distance (40 km / $80 \mathrm{~km}$ ) & $30 / 23$ \\
Race difficulty (easy/demanding) & $32 / 21$ \\
Average speed (< 14 km/h / $\geq 14 \mathrm{~km} / \mathrm{h}$ ) & $35 / 18$ \\
Races per horse (one / two / three / four races) & $8 / 15 / 5 / 0$ \\
\hline
\end{tabular}




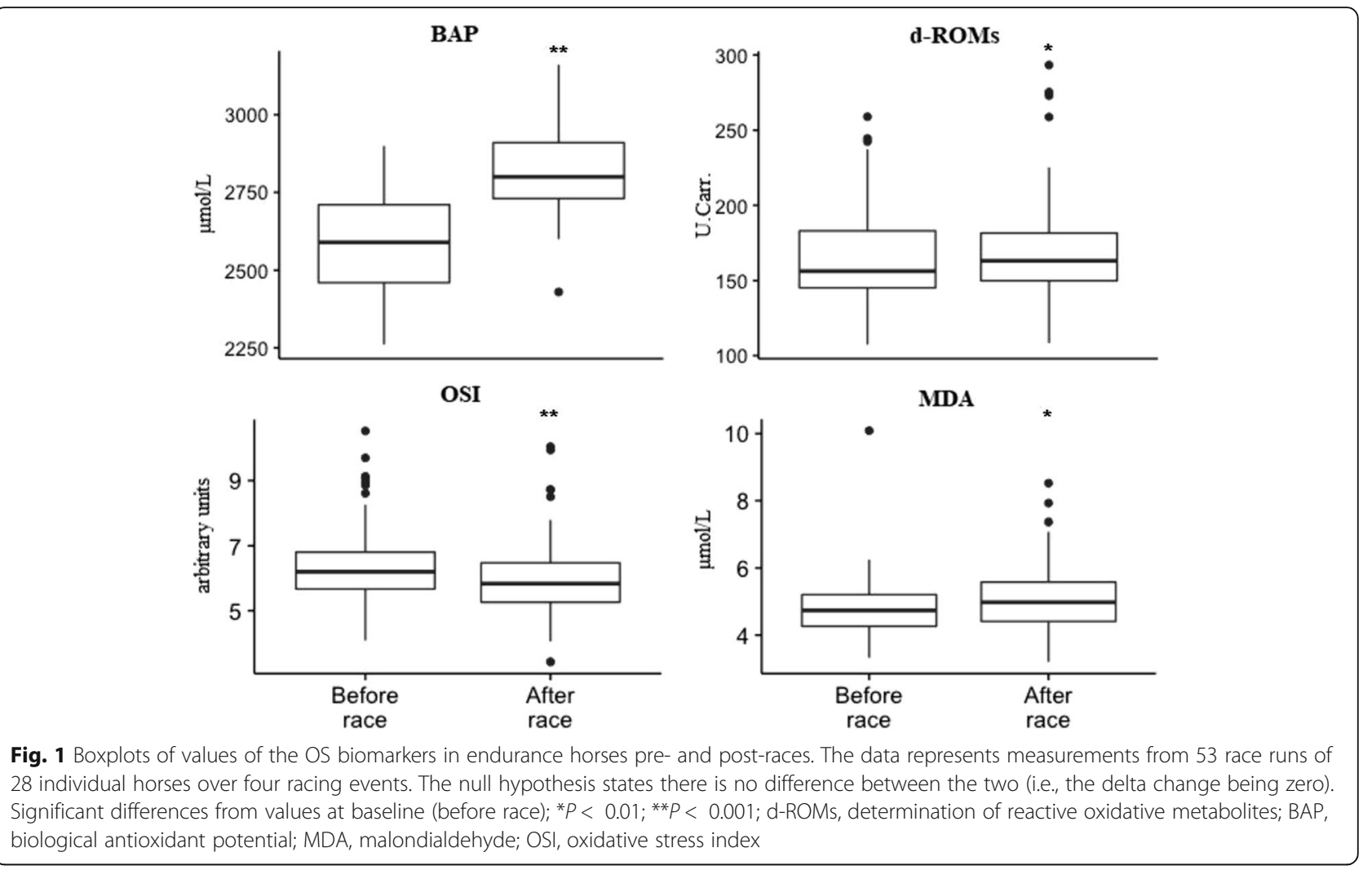

Table 2 Values of serum OS biomarkers in horses pre- and post-races by distance and difficulty

\begin{tabular}{|c|c|c|c|c|c|}
\hline \multirow[t]{2}{*}{ Parameter (unit) } & & \multicolumn{2}{|l|}{ Distance } & \multicolumn{2}{|l|}{ Race difficulty } \\
\hline & & $40 \mathrm{~km}$ & $80 \mathrm{~km}$ & Easy & Demanding \\
\hline \multirow[t]{4}{*}{ d-ROMs (U.Carr.) } & Pre-race & $164.8 \pm 7.6$ & $168.7 \pm 9.9$ & $166.2 \pm 6.8$ & $166.2 \pm 8.2$ \\
\hline & Post-race & $173.8 \pm 8.6$ & $174.2 \pm 11.3$ & $171.9 \pm 7.8$ & $172.3 \pm 8.4$ \\
\hline & $P$ & 0.002 & 0.2 & 0.04 & 0.03 \\
\hline & $P^{a}$ & 0.5 & & 0.9 & \\
\hline \multirow[t]{4}{*}{ BAP $(\mu \mathrm{mol} / \mathrm{L})$} & Pre-race & $2610.9 \pm 33.2$ & $2533.1 \pm 30.5$ & $2612.7 \pm 32.2$ & $2541.9 \pm 32.2$ \\
\hline & Post-race & $2820.4 \pm 28.2$ & $2840.4 \pm 31.4$ & $2817.5 \pm 28.8$ & $2840.5 \pm 22.8$ \\
\hline & $P$ & $<0.001$ & $<0.001$ & $<0.001$ & $<0.001$ \\
\hline & $P^{a}$ & 0.01 & & 0.02 & \\
\hline \multirow[t]{4}{*}{$\mathrm{MDA}(\mu \mathrm{mol} / \mathrm{L})$} & Pre-race & $5.1 \pm 0.2$ & $4.5 \pm 0.1$ & $4.7 \pm 0.1$ & $5.1 \pm 0.3$ \\
\hline & Post-race & $5.3 \pm 0.2$ & $4.7 \pm 0.2$ & $5.1 \pm 0.2$ & $5.1 \pm 0.3$ \\
\hline & $\mathrm{P}$ & 0.1 & 0.02 & 0.003 & 0.7 \\
\hline & $P^{a}$ & 0.9 & & 0.1 & \\
\hline \multirow[t]{4}{*}{ OSI (arbitrary units) } & Pre-race & $6.3 \pm 0.3$ & $6.7 \pm 0.4$ & $6.4 \pm 0.3$ & $6.6 \pm 0.3$ \\
\hline & Post-race & $6.1 \pm 0.3$ & $6.2 \pm 0.4$ & $6.1 \pm 0.3$ & $6.1 \pm 0.3$ \\
\hline & $P$ & 0.004 & $<0.001$ & 0.005 & $<0.001$ \\
\hline & $P^{a}$ & 0.01 & & 0.1 & \\
\hline
\end{tabular}

d-ROMs determination of reactive oxidative metabolites, BAP biological antioxidant potential, MDA malondialdehyde, OSI oxidative stress index P-significance for the differences within each category division (e.g. change in values at $40 \mathrm{~km}$ and at $80 \mathrm{~km}$ separately)

$\mathrm{P}^{\mathrm{a}}$-significance for the difference between the two categories (e.g. change in values at $40 \mathrm{~km}$ vs. at $80 \mathrm{~km}$ ) 
Table 3 Values of serum OS biomarkers in horses pre- and post-races by average speed and gender

\begin{tabular}{|c|c|c|c|c|c|}
\hline \multirow[t]{2}{*}{ Parameter (unit) } & & \multicolumn{2}{|c|}{ Average speed } & \multicolumn{2}{|l|}{ Gender } \\
\hline & & $<14 \mathrm{~km} / \mathrm{h}$ & $\geq 14 \mathrm{~km} / \mathrm{h}$ & Female & Male \\
\hline \multirow[t]{4}{*}{ d-ROMs (U.Carr.) } & Pre-race & $166.5 \pm 7.3$ & $160.6 \pm 8.2$ & $171.1 \pm 8.0$ & $164.1 \pm 11.9$ \\
\hline & Post-race & $171.5 \pm 8.2$ & $170.1 \pm 8.9$ & $177.2 \pm 9.8$ & $170.2 \pm 11.8$ \\
\hline & $P$ & 0.08 & 0.002 & 0.03 & 0.03 \\
\hline & $P^{a}$ & 0.3 & & 0.8 & \\
\hline \multirow[t]{4}{*}{ BAP $(\mu \mathrm{mol} / \mathrm{L})$} & Pre-race & $2570.2 \pm 28.4$ & $2585.4 \pm 39.6$ & $2570.8 \pm 29.82$ & $2588.5 \pm 46.9$ \\
\hline & Post-race & $2849.2 \pm 23.3$ & $2787.5 \pm 41.0$ & $2816.3 \pm 21.3$ & $2825.2 \pm 51.5$ \\
\hline & $P$ & $<0.001$ & $<0.001$ & $<0.001$ & $<0.001$ \\
\hline & $P^{a}$ & 0.1 & & 0.9 & \\
\hline \multirow[t]{4}{*}{ MDA $(\mu \mathrm{mol} / \mathrm{L})$} & Pre-race & $4.7 \pm 0.1$ & $5.2 \pm 0.3$ & $4.8 \pm 0.1$ & $4.9 \pm 0.3$ \\
\hline & Post-race & $5.0 \pm 0.2$ & $5.3 \pm 0.3$ & $5.1 \pm 0.1$ & $5.1 \pm 0.4$ \\
\hline & $P$ & 0.003 & 0.3 & 0.01 & 0.2 \\
\hline & $P^{a}$ & 0.8 & & 0.9 & \\
\hline \multirow[t]{4}{*}{ OSI (arbitrary units) } & Pre-race & $6.5 \pm 0.3$ & $6.2 \pm 0.3$ & $6.7 \pm 0.4$ & $6.4 \pm 0.5$ \\
\hline & Post-race & $6.04 \pm 0.3$ & $6.1 \pm 0.4$ & $6.4 \pm 0.5$ & $6.4 \pm 0.5$ \\
\hline & $P$ & $<0.001$ & 0.116 & $<0.001$ & $<0.001$ \\
\hline & $P^{a}$ & 0.02 & & 0.6 & \\
\hline
\end{tabular}

$d$-ROMs determination of reactive oxidative metabolites, BAP biological antioxidant potential, MDA malondialdehyde, OSI oxidative stress index P-significance for the differences within each category division (e.g. change in values at female and at male horses separately)

$\mathrm{P}^{\mathrm{a}}$-significance for the difference between the two categories (e.g. change in values at female vs. male horses)

only after easy races. Dividing the horse population in accordance with the average speed obtained, the changes in levels of d-ROMs increased significantly after the race only in horses of the faster speed category, levels of BAP increased significantly in both speed categories, and the concentration of MDA and values of OSI had significant changes only in the slower speed category. Both female and male horses showed significant differences in values of d-ROMs, BAP, and OSI after the race, compared to the values before the race. However, a significant change in concentrations of MDA due to race has been noted just in female horses. Both race distance and difficulty had a significant association with the change of BAP. The changes in values of OSI before and after the race have been influenced by race distance and average speed of horses.

Table 4 presents values of different OS markers in relation to each of the four races. Values of d-ROMs showed significant differences before and after the race during $40 \mathrm{~km}$ at the first race. With regard to values of BAP, significant differences before and after the race values have been noted in all but the second race. In addition, the changes in values of BAP were more pronounced after $80 \mathrm{~km}$ than after $40 \mathrm{~km}$ races at the third and fourth race events. Values of OSI showed significant differences before and after the race during $80 \mathrm{~km}$ at the third and after $40 \mathrm{~km}$ at the fourth race event.

\section{Discussion}

The intense exercise during endurance races in this study induced OS in horses demonstrated by increased values of d-ROMs, MDA, and BAP, and a decreased values of OSI. The increase in values of d-ROMs and MDA in horses undergoing endurance races could be attributed to the enhanced production of free radicals and lipid peroxidation resulting in oxidative damage $[6$, 22]. On the other hand, the rise in levels of BAP after the race indicates a mobilization of antioxidants as a response to an increased amount of ROS [6]. Oxidative stress depends on the balance between ROS and body's antioxidant system [23], therefore, OSI could more accurately reflect OS status due to a joint measure of oxidants and antioxidants properties. In the present study, a significant decrease in values of OSI shown after the race indicates a pronounced antioxidant response induced by endurance exercise.

Previous studies have evaluated OS in response to endurance exercise. Endurance race of $80 \mathrm{~km}$ caused an effect on oxidative status balance characterised by an increase in values of LPO, GSH and a decrease in values of GPx and TOC [17]. A study [18] reported OS after $80 \mathrm{~km}$ endurance race by an increase in total plasma LPO. On the other hand, changes in oxidative status markers (lipid hydroperoxides oxygen radical absorbance capacity, vitamin E, and GSH) have not been observed after $80 \mathrm{~km}$ endurance race [24]. The discrepancy between the mentioned findings [24] and the present 
Table 4 Values of serum OS biomarkers in horses pre- and post-races by separate race events

\begin{tabular}{|c|c|c|c|c|c|c|c|c|c|}
\hline \multicolumn{2}{|l|}{ Parameter (unit) } & \multicolumn{2}{|l|}{ First race } & \multicolumn{2}{|l|}{ Second race } & \multicolumn{2}{|l|}{ Third race } & \multicolumn{2}{|l|}{ Fourth race } \\
\hline & & $40 \mathrm{~km}(n=12)$ & $80 \mathrm{~km}(n=7)$ & $40 \mathrm{~km}(n=2)$ & $80 \mathrm{~km}(n=3)$ & $40 \mathrm{~km}(n=8)$ & $80 \mathrm{~km}(n=8)$ & $40 \mathrm{~km}(n=8)$ & $80 \mathrm{~km}(n=5)$ \\
\hline \multirow{4}{*}{$\begin{array}{l}\text { d-ROMs } \\
\text { (U.Carr.) }\end{array}$} & Pre-race & $160.9 \pm 11.8$ & $172.7 \pm 16.8$ & $163.8 \pm 13.5$ & $184.9 \pm 27.4$ & $162.7 \pm 9.2$ & $163.3 \pm 17.5$ & $174.9 \pm 6.2$ & $157.2 \pm 12.1$ \\
\hline & Post-race & $176.0 \pm 13.2$ & $175.6 \pm 20.2$ & $161.7 \pm 0.7$ & $184.2 \pm 22.0$ & $169.1 \pm 8.9$ & $173.8 \pm 19.7$ & $173.0 \pm 8.2$ & $156.7 \pm 14.7$ \\
\hline & P & $<0.001$ & 0.7 & 0.9 & 0.9 & 0.06 & 0.07 & 0.7 & 0.9 \\
\hline & $P^{a}$ & 0.07 & & 0.9 & & 0.5 & & 0.9 & \\
\hline \multirow[t]{4}{*}{ BAP ( $\mu \mathrm{mol} / \mathrm{L})$} & Pre-race & $2575.8 \pm 51.6$ & $2628.6 \pm 71.9$ & $2820.0 \pm 40.0$ & $2573.3 \pm 53.6$ & $2556.3 \pm 52.6$ & $2446.3 \pm 21.5$ & $2692.5 \pm 34.3$ & $2516.0 \pm 53.5$ \\
\hline & Post-race & $2806.7 \pm 49.0$ & $2837.1 \pm 90.3$ & $2905.0 \pm 155.0$ & $2883.3 \pm 69.4$ & $2812.5 \pm 33.6$ & $2836.3 \pm 32.3$ & $2818.8 \pm 29.3$ & $2826.0 \pm 65.7$ \\
\hline & $P$ & $<0.001$ & 0.04 & 0.7 & 0.06 & $<0.001$ & $<0.001$ & $<0.01$ & $<0.01$ \\
\hline & $P^{a}$ & 0.8 & & 0.3 & & 0.03 & & 0.02 & \\
\hline \multirow{4}{*}{$\begin{array}{l}\mathrm{MDA} \\
(\mu \mathrm{mol} / \mathrm{L})\end{array}$} & Pre-race & $4.9 \pm 0.2$ & $4.5 \pm 0.2$ & $8.2 \pm 1.9$ & $3.8 \pm 0.2$ & $5.2 \pm 0.2$ & $4.6 \pm 0.1$ & $4.8 \pm 0.1$ & $4.3 \pm 0.2$ \\
\hline & Post-race & $4.9 \pm 0.2$ & $4.9 \pm 0.3$ & $7.8 \pm 0.7$ & $3.5 \pm 0.1$ & $5.3 \pm 0.2$ & $4.8 \pm 0.2$ & $5.7 \pm 0.4$ & $5.2 \pm 0.3$ \\
\hline & P & 0.8 & 0.2 & 0.8 & 0.08 & 0.5 & 0.5 & 0.08 & 0.02 \\
\hline & $P^{a}$ & 0.3 & & 0.9 & & 0.9 & & 0.9 & \\
\hline \multirow{4}{*}{$\begin{array}{l}\text { OSI } \\
\text { (arbitraryunits) }\end{array}$} & Pre-race & $6.2 \pm 0.4$ & $6.7 \pm 0.8$ & $5.8 \pm 0.4$ & $7.1 \pm 0.9$ & $6.4 \pm 0.4$ & $6.7 \pm 0.7$ & $6.5 \pm 0.3$ & $6.2 \pm 0.4$ \\
\hline & Post-race & $6.2 \pm 0.4$ & $6.3 \pm 0.9$ & $5.6 \pm 0.3$ & $6.4 \pm 0.7$ & $6.0 \pm 0.3$ & $6.1 \pm 0.6$ & $6.1 \pm 0.3$ & $5.5 \pm 0.5$ \\
\hline & P & 0.9 & 0.07 & 0.2 & 0.07 & 0.08 & 0.02 & 0.01 & 0.2 \\
\hline & $P^{a}$ & 0.05 & & 0.1 & & 0.4 & & 0.4 & \\
\hline
\end{tabular}

$d$-ROMs determination of reactive oxidative metabolites, BAP biological antioxidant potential, MDA malondialdehyde, OSI oxidative stress index P-significance for the differences within each category division (e.g. change in pre-race and post-race values)

$\mathrm{P}^{\mathrm{a}}$-significance for the difference between the two categories (e.g. change in values at $40 \mathrm{~km} \mathrm{vs} .80 \mathrm{~km}$ )

study could be attributed to OS biomarker selection, a small number of horses (four), and a relatively low average speed of $10.5 \mathrm{~km} / \mathrm{h}$ in the former compared to the present study which had a larger sample size and horses competing with almost 30\% faster average speed. A previous report [2] assessed the levels of SOD, GPx, reduced GSH, catalase, and the plasma MDA in endurance horses, performing at different distances. Interestingly, that study did not record any significant changes in values of OS biomarkers after the race, although higher values of SOD, GPx, GSH, and MDA were recorded after the $80 \mathrm{~km}$ race but not at $160 \mathrm{~km}$. These results could also be attributed to the relatively small number (15) of studied horses and potentially individual differences in measured OS biomarkers could have precluded the true association of OS biomarkers and race distance. As the effect of individual horses could vary between OS biomarkers, the selection of the markers should be an important aspect for consistent results. The results of the present study showed only BAP not to have a significant horse effect, hence, making it a more consistent OS biomarker than d-ROMs, MDA, and OSI. In this study d-ROMs, MDA, and OSI showed discordant changes in approximately one-third of the horses. These findings indicate that there are other determinants and explanatory variables also influencing the changes in d-ROMs, MDA, and OSI that have not been followed in this study. Similarly, a report [25] noted fluctuation of values of d-ROMs especially, but also of BAP to a lesser degree, in ten Thoroughbred horses. Studies involving a large number of horses are needed in order to better understand the physiological levels and changes of OS biomarkers.

In the present study, MDA has also shown increased concentrations after the race indicating a potential cumulative cell membrane lipid peroxidation. A rise in erythrocyte MDA concentration was also recorded after short-term heavy exercise in jumping horses [22], as well as by an increased intensity ride in ten Maremmana stallions [26]. A previous study [27] reported significant changes of TBARS, a test that measures mainly but not only MDA, induced at $80 \mathrm{~km}$ endurance race, but not in horses competing at $160 \mathrm{~km}$. That study suggested horses competing at higher speed (which occurred at $80 \mathrm{~km}$ races) are more prone to rise in TBARS levels after exercise. Contrary, a study on five horses competing at $160 \mathrm{~km}$ race, noted an increase in values of TBARS after the race [14]. In the present study, an increased concentration of MDA was noted only in horses at $80 \mathrm{~km}$ races with no significant difference in average speed between race distances thus, it is not possible to confirm the suggestion of influence of speed [27]. Lack of increased lipid peroxidation after half race distance and an increase after $80 \mathrm{~km}$ endurance race have also been noted by measuring values of plasma LPO [17]. Actually, the present study showed a significant rise in the concentration of MDA after the race only at slower average speed. Furthermore, the present study also adds race difficulty as another factor potentially influencing changes of MDA, as a significant change was shown only at easy races. These unexpected results raise suspicion for a potential individual 
variation in lipid peroxidation between horses and the possibility that training status or other parameters not followed in this study, could influence concentrations of MDA. On the other hand, lower values of LPO have been recorded in an easier race in comparison to a more difficult race as defined by the riders [17]. However, a clear definition of race difficulty could be challenging, especially considering possible subjectivity of riders opinion.

In previous studies of OS subsequent endurance races, opponent changes in antioxidant status have been noted. After $80 \mathrm{~km}$ race an increase in values of GSH $[17,18]$ and GPx $[15,18]$ has been noted, contrary, another study showed a decrease in values of GPx [17]. A study on 5 horses competing at $160 \mathrm{~km}$ endurance race noted a drop in values of TAS at $80 \mathrm{~km}$, but a rise of both values of SOD and TAS at the race end [14]. The inconsistent findings of previous studies could be attributed to the antioxidant biomarker selection, since some of the vast antioxidant biomarkers show lower antioxidant capacity caused by exercise, but simultaneously other biomarkers enhance their activity. Therefore we believe that BAP could be more effective in delineating general antioxidant status, since it provides an overall measurement of different antioxidants including uric acid, ascorbic acid, proteins, TOC, bilirubin and others [7]. The increase in levels of BAP after the race in the present study indicates an increase in the antioxidant potential as a consequence of exercise. It suggests that during prolonged exercise OS induces a response to the increase in ROS thus, results in an increase in levels of BAP. Similar findings have also been noted in Thoroughbred horses after a short-term maximum treadmill exercise [28]. In that study, the rise in BAP levels was more pronounced immediately after exercise, with a clear drop in levels of BAP 30 min after exercise. After finishing the race endurance horses have a maximum of $30 \mathrm{~min}$ rest before presenting to the final inspection. Owners did not allow blood sampling during that rest period in the present study since it could negatively affect the horse's attitude thus compromising the final results of the forthcoming mandatory veterinary inspection. Therefore, our sampling time was $30 \mathrm{~min}$ after the end of the race and it could have been the reason of our comparably lower recorded levels of BAP, although still significantly different from the values before the race. Similarly, in the study were samples have been taken after $80 \mathrm{~km}$ race as well as after $60 \mathrm{~min}$ of recovery period, there was an evident decrease in GPx levels during the last sampling [15]. Though, in the abovementioned study it is not defined if the samples have been taken immediately after finishing the race or after passing the mandatory veterinary inspection. The time preceding the veterinary inspection after each loop is very short (often lasting up to few minutes) and considerable effort is put on minimising any discomfort to horses. In the study on Thoroughbred horses undertaking treadmill exercise [28], an increase in levels of d-ROMs in response to exercise has been reported. Interestingly, in that study, at $30 \mathrm{~min}$ after exercise, the levels of d-ROMs returned to baseline. Therefore, the sampling time could have also influenced the results of d-ROMs in the present study. Similarly, in a previous study an effect of $3 \mathrm{~h}$ recovery period after $80 \mathrm{~km}$ endurance race on prooxidative biomarker values has been noted since values of LPO also decreased to baseline [17]. An overall increase in levels of d-ROMs in the present study has been noted after the race but, taking into account race distances, this increase was significant only in horses competing at shorter distances. Horses competing at $80 \mathrm{~km}$ races were running twice longer and furthermore had one more compulsory rest period according to endurance rules that could have influenced our results. In addition, the lack of the elevation of d-ROMs after $80 \mathrm{~km}$ races could also indicate that the horses used in this study had an adaptive antioxidant response. Horses competing at $80 \mathrm{~km}$ are trained for longer periods than those competing at $40 \mathrm{~km}$ what could induce adaptations to OS, supported by a study in Standardbred horses that proved that training increase antioxidant capacity [29].

In the present study, a decrease in values of OSI has been noted after the race similarly to a previous report on Thoroughbred horses [28] using the BAP/d-ROMs ratio, which is an inverse formula of the one used in the present study. Nevertheless, the results of both studies indicate a more pronounced antioxidant status as a result of OS induced by exercise. Reference ranges of d-ROMs, BAP and OSI values have been studied in 372 Thoroughbreds racehorses [12]. That study measured values of OS biomarkers only during resting and the levels of both BAP and d-ROMs OS markers have been very similar to our findings.

The observed changes in levels of BAP after exercise were associated with both race distance and difficulty with higher values recorded on longer and demanding races. These findings indicate that the type and intensity of exercise can also influence OS in endurance horses. In a previous study, comparing results between two $80 \mathrm{~km}$ races of unequal difficulties, a more pronounced rise in GSH has also been recorded after a more difficult race [17]. More pronounced changes in antioxidant biomarkers were noted in eventing horses competing at a higher intensity exercise indicating the influence of increased anaerobic and musculoskeletal demands [30]. Expectably, a more pronounced increase in levels of BAP coupled with a more pronounced decrease in values of OSI was recorded on longer compared to the shorter races, as well as on demanding compared to easy races, which could indicate a stronger antioxidant response due to a higher intensity of exercise. However, even when considering only horses participating at $40 \mathrm{~km}$ 
races an increase in values of BAP and OSI was evident after the race, which emphasises that horses competing at lower distances are also susceptible to OS, especially characterised by a pronounced activation of antioxidant defence mechanisms. Accordingly, OS could still have negative consequences on performance and health in shorter races.

Taking into account the speed of horses, more pronounced changes in values of OSI have been noted at slower average speed. Since less experienced horses often compete at a lower speed, this result could indicate that those horses are more susceptible to OS than more experienced horses. The same was proved in mice chronically exposed to exercise, that showed less oxidative damage after exhaustive exercise than the untrained animals. This is largely due to the upregulation of endogenous antioxidant enzymes such as mitochondrial SOD, GPx, and $\gamma$-glutamylcysteine synthetase [31]. On the other hand, more pronounced adaptation to OS in horses competing at higher speed could be explained by more intense regular training. An adaptive response to training has also been noted in serum amyloid A concentrations in endurance horses after training session resembling a race [32].

The results did not show gender to influence any studied OS markers, however, when separately evaluating female and male horses, only concentrations of MDA was significantly different from pre-race values in mares only. This could be a spurious finding due to a lesser number of male horses. A previous study on Warmblood horses noted increased SOD activities in response to exercise only in mares [33].

When comparing individually the 4 races results with the whole testing population, an inconsistency is present, especially in the second race. However, there were only 2 horses competing at $40 \mathrm{~km}$ and 3 horses competing at $80 \mathrm{~km}$ at the second race. Nevertheless, we find noteworthy mentioning that the BAP levels in all races, except the second race, have shown consistent results what additionally accentuates the reliability of this biomarker in representing antioxidative status in endurance horses.

There were some limitations of this study that need to be emphasised. Lack of standardised feeding or detailed nutritional surveys to determine the intake level of antioxidants can be considered a limitation. However, most of the horses were fed balanced formulas for endurance horses. In light of standardisation, as this study was not performed in an experimentally controlled setting, the heterogeneity of horses may have introduced bias or confounding of the results. Since all horses have been transported to the event from different locations, the transport per se could have also influenced the results despite that all the horses have been given a period of rest in proportion to the distance and stress caused by the transportation before the start of the competition.
However, it was taken that the transportation effect had ceased since all the animals have successfully passed the veterinary examination and were declared fit to compete prior to the blood sampling. Another limitation was that the enrolment into the study was voluntary and it did not include all the participating horses.

\section{Conclusions}

The submaximal endurance exercise-induced simultaneous changes in all measured OS markers in this study. Unlike d-ROMs, MDA, and OSI biomarkers, BAP was observed to have low individual variations between and within horses, making it the most reliable biomarker of OS in endurance horses in the present study. Our results indicate that disequilibrium between oxidants and antioxidants also occurred during shorter races and those performed on less demanding racetracks, both in particular characterised by a pronounced antioxidant defence.

\section{Abbreviations \\ BAP: Biological antioxidant potential; d-ROMs: Reactive oxygen metabolites; GPx: Glutathione peroxidase; GSH: Total glutathione; MDA: Malondialdehyde; OS: Oxidative stress; OSI: Oxidative stress index; ROS: Reactive oxygen species; TBARS: Total barbituric acid reactive substances}

\section{Availability of data and materials \\ The datasets used and/or analysed during the current study are available from the corresponding author on reasonable request.}

\section{Authors' contributions \\ NBB and ZV conceived and designed the study. NBB, JG, KK and ZV contributed in an equal manner to the acquisition of data. Jك̌ and DB: contributed in laboratory analysis. KB conceived the statistical analysis, analysed the data and contributed to the interpretation of the data. NBB was a major contributor in writing the manuscript. ZV supervised the manuscript. All of the authors were involved in drafting the article and revising it critically for important intellectual content. All authors read and approved the final manuscript.}

Ethics approval and consent to participate

A written consent statement was obtained from the owners of animals included in the study. The Ethical Board of the Faculty of Veterinary Medicine approved the study (File No. 640-01/16-17/75; Record No. 251-61-01/139-16-2).

\section{Consent for publication \\ Not applicable.}

\section{Competing interests}

The authors declare that they have no competing interests.

\section{Publisher's Note}

Springer Nature remains neutral with regard to jurisdictional claims in published maps and institutional affiliations.

\footnotetext{
Author details

${ }^{1}$ Clinic for Surgery, Orthopaedics and Ophthalmology, Faculty of Veterinary Medicine, University of Zagreb, Heinzelova 55, 10000 Zagreb, Croatia. ${ }^{2}$ Clinic for Internal Diseases, Faculty of Veterinary Medicine, University of Zagreb, Heinzelova 55, 10000 Zagreb, Croatia. ${ }^{3}$ Department of Pharmacology and Toxicology, Faculty of Veterinary Medicine, University of Zagreb, Heinzelova 55, 10000 Zagreb, Croatia. ${ }^{4}$ Department of Animal Nutrition and Dietetics, Faculty of Veterinary Medicine, University of Zagreb, Heinzelova 55, 10000 Zagreb, Croatia. ${ }^{5}$ Faculty of Veterinary Medicine, University of Zagreb, Heinzelova 55, 10000 Zagreb, Croatia. ${ }^{6}$ School of Veterinary Science, Massey University, Palmerston North, New Zealand. ${ }^{7}$ Department of Radiology,
} 
Ultrasound Diagnostic and Physical Therapy, Faculty of Veterinary Medicine, University of Zagreb, Heinzelova 55, 10000 Zagreb, Croatia.

Received: 8 February 2018 Accepted: 24 June 2018

Published online: 06 July 2018

\section{References}

1. Butler PJ, Woakes AJ, Smale K, Roberts CA, Hillidge CJ, Snow DH, Marlin DJ, Respiratory and cardiovascular adjustments during exercise of increasing intensity and during recovery in thoroughbred racehorses. J Exp Biol. 1993; 179:159-80.

2. Siqueira RF, Weigel RA, Nunes GR, Mori CS, Fernandes WR. Oxidative profiles of endurance horses racing different distances. Ara Bras Med Vet Zootec. 2014;66:455-61.

3. Sjodin B, Hellsten Westing Y, Apple FS. Biochemical mechanisms for oxygen free radical formation during exercise. Sports Med. 1990;10:236-54.

4. Sies H. Oxidative stress: from basic research to clinical application. Am J Med. 1991:91:31S-8S.

5. Williams CA. The effect of oxidative stress during exercise in the horse. J Anim Sci. 2016:94:4067-75.

6. Celi $P$, Sullivan M, Evans D. The stability of the reactive oxygen metabolites (d-ROMs) and biological antioxidant potential (BAP) tests on stored horse blood. Vet J. 2010;183:217-8.

7. Benzie IFF, Strain JJ. The ferric reducing ability of plasma (FRAP) as a measure of "antioxidant power": the FRAP assay. Anal Biochem. 1996;239:70-6.

8. Grotto D, Santa Maria LD, Boeira S, Valentini J, Char ME, Moro AM, Nascimento PC, Pomblum VJ, Garcia SC. Rapid quantification of malondialdehyde in plasma by high performance liquid chromatography visible detection. J Pharm Biomed Anal. 2007;43:619-24.

9. Esterbauer H, Schaur RJ, Zollner H. Chemistry and biochemistry of 4hydroxynonenal, malonaldehyde and related aldehydes. Free Radic Biol Med. 1991;11:81-128.

10. Lee S, Hashimoto J, Suzuki T, Satoh A. The effects of exercise load during development on oxidative stress levels and antioxidant potential in adulthood. Free Radic Res. 2017:51:179-86.

11. Po E, Williams C, Muscatello G, Celi P. Assessment of oxidative stress biomarkers in exhaled breath condensate and blood of thoroughbred foals. Vet J. 2013;196:269-71

12. Kusano K, Yamazaki M, Kiuchi M, Kaneko K, Koyama K. Reference range of blood biomarkers for oxidative stress in thoroughbred racehorses ( $2-5$ years old). J Equine Vet Sci. 2016:27:125-9.

13. Tsuzuki N, Sasaki N, Kusano K, Endo Y, Torisu S. Oxidative stress markers in thoroughbred horses after castration surgery under inhalation anesthesia. J Equine Vet Sci. 2016;27:77-9.

14. Frankiewicz-Jozko A, Szarska E. Anti-oxidant level to exercise in the blood of endurance horses. Biol Sport. 2000;17:217-27.

15. Hargreaves BJ, Kronfeld DS, Waldron JN, Lopes MA, Gay LS, Saker KE, Cooper WL, Sklan DJ, Harris PA. Antioxidant status and muscle cell leakage during endurance exercise. Equine Vet J. 2002;34:116-21.

16. Marlin DJ, Fenn K, Smith N, Deaton CD, Roberts CA, Harris PA, Dunster C, Kelly FJ. Changes in circulatory antioxidant status in horses during prolonged exercise. J Nutr. 2002;132(Suppl 2):1622-7.

17. Williams CA, Kronfeld DS, Hess TM, Saker KE, Waldron JE, Crandell KM, Harris PA. Comparison of oxidative stress and antioxidant status in endurance horses in three 80-km races. Equine Comp Exerc Physiol. 2005;2:153-7.

18. Williams CA, Kronfeldt DS, Hess TM, Saker KE, Waldron JN, Crandell KM, Hoffman RM, Harris PA. Antioxidant supplementation and subsequent oxidative stress of horses during an 80-km endurance race. J Anim Sci. 2004; 82:588-94.

19. Bergero D, Miraglia N, Schiavone A, Polidori M, Prola L. Effect of dietary polyunsaturated fatty acids and vitamin $\mathrm{E}$ on serum oxidative status in horses performing very light exercise. Ital J Anim Sci. 2004;3:141-5.

20. Team RDC. R: a language and environment for statistical computing. Vienna: R Foundation for Statistical Computing; 2015.

21. Bates $D$, Maechler $M$, Bolker B, Walker S. Fitting linear mixed-effects models using Ime4. J Stat Softw. 2015;67:1-48.

22. Soares JCM, Zanella R, Bondan C, Alves LP, de Lima MR, da Motta AC, Zanella EL. Biochemical and antioxidant changes in plasma, serum, and erythrocytes of horses before and after a jumping competition. J Equine Vet Sci. 2011;31:357-60.
23. McBride JM, Kraemer WJ. Free radicals, exercise, and antioxidants. J Str Cond Res. 1999:13:175-83

24. Kinnunen S, Atalay M, Hyyppä S, Lehmuskero A, Hänninen O, Oksala N. Effects of prolonged exercise on oxidative stress and antioxidant defence in endurance horse. J Sports Sci Med. 2005;4:415-21.

25. Tsuzuki N, Endo Y, Kikkawa L, Korosue K, Kaneko Y, Kitauchi A, Katamoto H, Hidaka Y, Hagio M, Torisu S. Effects of ozonated autohemotherapy on the antioxidant capacity of thoroughbred horses. J Vet Med Sci. 2015;77:1647-50

26. Chiaradia E, Avellini L, Rueca F, Spaterna A, Porciello F, Antonioni MT, Gait A. Physical exercise, oxidative stress and muscle damage in racehorses. Comp Biochem Physiol B Biochem Mol Biol. 1998;119:833-6.

27. Holbrook TC, McFarlane D, Schott HC. Neuroendocrine and nonneuroendocrine markers of inflammation associated with performance in endurance horses. Equine Vet J. 2010;42:123-8.

28. Tsubone $\mathrm{H}$, Hanafusa $\mathrm{M}$, Endo M, Manabe $\mathrm{N}$, Hiraga A, Ohmura $\mathrm{H}$, Aida $\mathrm{H}$. Effect of treadmill exercise and hydrogen-rich water intake on serum oxidative and anti-oxidative metabolites in serum of thoroughbred horses. J Equine Vet Sci. 2013;24:1-8.

29. Janiak M, Suska M, Dudzińska W, Skotnicka E. Blood glutathione status and activity of glutathione-metabolizing antioxidant enzymes in erythrocytes of young trotters in basic training. J Anim Physiol Anim Nutr. 2010;94:137-45.

30. Williams CA, Burk AO. Antioxidant status in elite three-day event horses during competition. Oxid Med Cell Long. 2012;2012: Article ID 572090, 8 pages. https://doi.org/10.1155/2012/572090.

31. Salminen A, Vihko V. Lipid peroxidation in exercise myopathy. Exp Mol Pathol. 1983:38:380-8.

32. Cywinska A, Witkowski L, Szarska E, Schollenberger A, Winnicka A. Serum amyloid a (SAA) concentration after training sessions in Arabian race and endurance horses. BMC Vet Res. 2013;9:91.

33. Andriichuk A, Tkachenko H, Kurhaluk N. Gender differences of oxidative stress biomarkers and erythrocyte damage in well-trained horses during exercise. J Equine Vet Sci. 2014;34:978-85.

\section{Ready to submit your research? Choose BMC and benefit from:}

- fast, convenient online submission

- thorough peer review by experienced researchers in your field

- rapid publication on acceptance

- support for research data, including large and complex data types

- gold Open Access which fosters wider collaboration and increased citations

- maximum visibility for your research: over $100 \mathrm{M}$ website views per year

At BMC, research is always in progress.

Learn more biomedcentral.com/submissions 